\title{
EFFECT OF RUGOSITY ON A FLOW GOVERNED BY STATIONARY NAVIER-STOKES EQUATIONS
}

\author{
BY \\ YOUCEF AMIRAT. DIDIER BRESCH. JÉROOME LEMIOINE. AND JACQUES SIMION \\ Laboratoire de Mathématiques Appliquées, CNRS (UMR 6620), Université Blaise Pascal \\ (Clermont-Ferrand 2), 63177 Aubière cedex, France
}

\begin{abstract}
We study the stationary flow of a fluid occupying a 3D infinite horizontal domain bounded by a rough wall that is at rest and by a plane that moves with a constant velocity. The rough wall is a plane covered with periodically distributed asperities of size $\varepsilon$. We prove that, outside a neighbourhood of the rough region, the flow behaves asymptotically as a Couette flow, as $\varepsilon \rightarrow 0$, up to an exponentially small error.
\end{abstract}

1. Introduction. Numerical simulation of flows on rough walls, riblets or waves, for example, requires wall laws. These wall laws are boundary conditions that are imposed on a fictive flat wall inside the domain in order to get rid of the region with strong gradient that contains rugosity by taking into account their effect on the flow. Such simulations can be found in [1], [2], [7], for example. Actually, these wall laws come from empirical logarithmic calculations; see, for instance, [9], [12]. This motivates the mathematical study of the effect of rugosity in some simplified situations in order to see how such laws could be rigorously derived.

Here, we consider a viscous incompressible fluid occupying a 3D infinite horizontal domain bounded by two walls, a plane one and a rough one, which is a plane covered with periodically distributed rugosities. The rough wall is assumed to be at rest and the plane one moves with a constant horizontal velocity. We prove the existence of a Couette flow, depending on the size $\varepsilon$ of the asperities, which approximates the stationary Navier-Stokes flow outside the rough region, up to an error decreasing exponentially fast with respect to $\varepsilon$. A similar result is established in [5] for a Stokes flow, with an explicitly calculated Couette approximation. The extra difficulty here is that, because of nonlinearity, we are no longer able to perform such an explicit calculation.

More precisely, we prove that the velocity is the sum of three terms: a Couette flow $x_{3} / l_{3}$ where $x_{3}$ is the vertical coordinate and $l_{3}$ is the thickness of the flow; another Couette flow $\varepsilon\left(x_{3} / l_{3}-1\right) f_{\varepsilon}$ where $f_{\varepsilon}$ is a constant vector; a remainder $w_{\varepsilon}$ that is bounded outside a neighbourhood of the rough wall by $\left|w_{\varepsilon}(x)\right| \leq c \exp \left(-c^{\prime} x_{3} / \varepsilon\right)$.

Received December 13, 1999.

2000 Mathematics Subject Classification. Primary 49J20, 35Q30.

Key words and phrases. Navier-Stokes equations, asymptotic behaviour, rugosity. 
In order to prove this result, we write the equations satisfied by $w_{\varepsilon}$ for an arbitrary given $f_{\varepsilon}$. Then, we approximate $w_{\varepsilon}$ by a solution $\Psi_{\varepsilon}$ of Navier-Stokes type equations in a half-space. Such a solution has a limit $\bar{\Psi}_{\varepsilon \infty}$ as $x_{3} \rightarrow \infty$ and the convergence is controlled by $c \exp \left(-c^{\prime} x_{3} / \varepsilon\right)$. Finally, we prove that $f_{\varepsilon}$ may be chosen such that $\bar{\Psi}_{\varepsilon \infty}=0$ by using a fixed point argument. This gives the result.

The exponential control of the convergence relies on a Saint-Venant type estimate established in [4]. For similar estimates, see [10], [11], [14], and [15] and references cited therein. For other studies of rugosity in problems governed by various pde's, see [6], [8].

2. Main result. Let us consider a viscous fluid occupying an infinite horizontal domain bounded by two walls, a plane one $\mathcal{P}$ and a rough one $\mathcal{R}_{\varepsilon}$, covered with periodically distributed asperities of small size $\varepsilon$. The plane $\mathcal{P}$ is moving in a parallel direction to $\mathcal{R}_{\varepsilon}$ with a constant velocity $g$. The domain of the flow is

$$
\mathcal{O}_{\varepsilon}=\left\{x \in \mathbb{R}^{3}: x^{\prime} \in \mathbb{R}^{2}, \eta_{\varepsilon}\left(x^{\prime}\right)<x_{3}<l_{3}\right\}
$$

where $x^{\prime}=\left(x_{1}, x_{2}\right), l_{3}>0, \varepsilon>0$ and $\eta_{\varepsilon}$ (representing the profile of asperities) is the function given by

$$
\eta_{\varepsilon}\left(x^{\prime}\right)=\varepsilon \eta\left(\frac{x^{\prime}}{\varepsilon}\right),
$$

$\eta$ being periodic with respect to $x_{1}$ and $x_{2}$ with periods $l_{1}$ and $l_{2}$. The velocity $u_{\varepsilon}=$ $\left(u_{\varepsilon 1}, u_{\varepsilon 2}, u_{\varepsilon 3}\right)$ and the pressure $p_{\varepsilon}$ satisfy the stationary Navier-Stokes equations

$$
-\nu \Delta u_{\varepsilon}+u_{\varepsilon} \cdot \nabla u_{\varepsilon}+\nabla p_{\varepsilon}=0, \quad \nabla \cdot u_{\varepsilon}=0,\left.\quad u_{\varepsilon}\right|_{\mathcal{R}_{\varepsilon}}=0,\left.\quad u_{\varepsilon}\right|_{\mathcal{P}}=g,
$$

where $\nu>0$ and $g=\left(g^{\prime}, 0\right)$ are constant, and they are assumed to be periodic with respect to $x_{1}$ and $x_{2}$ with periods $\varepsilon l_{1}$ and $\varepsilon l_{2}$. We assume $1 / \varepsilon$ to be an integer so that $\eta_{\varepsilon}, u_{\varepsilon}$ and $p_{\varepsilon}$ are also periodic with respect to $x_{1}$ and $x_{2}$ with periods $l_{1}$ and $l_{2}$. Setting $S_{\varepsilon}=\left(0, \varepsilon l_{1}\right) \times\left(0, \varepsilon l_{2}\right)$, we denote by

$$
\Omega_{\varepsilon}=\left\{x \in \mathbb{R}^{3}: x^{\prime} \in S_{\varepsilon}, \eta_{\varepsilon}\left(x^{\prime}\right)<x_{3}<l_{3}\right\}
$$

the bounded part of the domain that is bounded by the part of the rough wall $R_{\varepsilon}=$ $\left\{x: x^{\prime} \in S_{\varepsilon}, x_{3}=\eta_{\varepsilon}\left(x^{\prime}\right)\right\}$, by the part of plane $P=\left\{x: x^{\prime} \in S_{\varepsilon}, x_{3}=l_{3}\right\}$, and by the immaterial lateral boundary $L_{\varepsilon}=\left\{x: x^{\prime} \in \partial S_{\varepsilon}, \eta_{\varepsilon}\left(x^{\prime}\right) \leq x_{3} \leq l_{3}\right\}$. Clearly, $\mathcal{O}_{\varepsilon}$ is generated by periodic horizontal translations of $\Omega_{\varepsilon}$. We denote, for $m \geq 0$,

$$
\begin{aligned}
H_{\mathrm{per}}^{m}\left(\Omega_{\varepsilon}\right)=\left\{v \in H_{\mathrm{loc}}^{m}\left(\mathcal{O}_{\varepsilon}\right):\right. & v \in H^{m}\left(\Omega_{\varepsilon}\right), \\
& \left.v\left(x_{1}, x_{2}, x_{3}\right)=v\left(x_{1}+\varepsilon l_{1}, x_{2}, x_{3}\right)=v\left(x_{1}, x_{2}+\varepsilon l_{2}, x_{3}\right)\right\},
\end{aligned}
$$

provided with the norm of $H^{m}\left(\Omega_{\varepsilon}\right)$.

The existence of a velocity $u_{\varepsilon}$ in this space can be proved by using a Hopf divergence free extension of the boundary data, as in Section 7.1, p. 101 of [13]. Here such an extension is explicitly given by $s\left(x_{3}\right) g$ provided that $s\left(l_{3}\right)=1$ and $s$ vanishes outside a neighbourhood of $l_{3}$. The pressure $p_{\varepsilon}$ is then obtained by the de Rham Theorem. Its periodicity may be proved as in Theorem 1 of [4]. In this way, we get the following existence result. 
Proposition 1. There exists a solution of (1) such that

$$
u_{\varepsilon} \in\left(H_{\mathrm{per}}^{1}\left(\Omega_{\varepsilon}\right)\right)^{3}, \quad p_{\varepsilon} \in L_{\mathrm{per}}^{2}\left(\Omega_{\varepsilon}\right), \quad \int_{\Omega_{\varepsilon}} p_{\varepsilon}=0 .
$$

Moreover, for $\varepsilon$ small enough, such a solution is unique.

We are interested in the asymptotic behaviour, as $\varepsilon \rightarrow 0$, of this solution. Our objective is to show that, outside a neighbourhood of the rough wall, the flow behaves asymptotically as a Couette flow and more precisely that

$$
u_{\varepsilon}(x)=\frac{x_{3}}{l_{3}} g+\varepsilon\left(\frac{x_{3}}{l_{3}}-1\right) f_{\varepsilon}+w_{\varepsilon}(x),
$$

where $f_{\varepsilon}=\left(f_{\varepsilon 1}, f_{\varepsilon 2}, 0\right)$ is a vector of $\mathbb{R}^{3}$ and $w_{\varepsilon}(x)$ decays exponentially with respect to $\varepsilon$. Denoting $\bar{\eta}=\sup \left\{\eta\left(x^{\prime}\right): x^{\prime} \in \mathbb{R}^{2}\right\}$, the main result is the following one.

Theorem 2. Let $\left(u_{\varepsilon}, p_{\varepsilon}\right)$ satisfy (1) and (2). There exist two positive numbers $c$ and $c^{\prime}$ and, for any $\alpha \in \mathbb{N}^{3}$, a positive number $c_{\alpha}^{\prime \prime}$, such that: for any $\varepsilon<c$, there exists $f_{\varepsilon}=\left(f_{\varepsilon 1}, f_{\varepsilon 2}, 0\right)$ such that the remainder $w_{\varepsilon}$, defined by $(3)$, and $p_{\varepsilon}$ satisfy

$$
\left|\partial^{\alpha} w_{\varepsilon}(x)\right|+\left|\partial^{\alpha} p_{\varepsilon}(x)\right| \leq c_{\alpha}^{\prime \prime} \exp \left(-c^{\prime} \frac{x_{3}}{\varepsilon}\right)
$$

for any $x \in \Omega_{\varepsilon}$ such that $x_{3} \geq \varepsilon(\bar{\eta}+1)$.

Remark that $f_{\varepsilon}$ is not unique since the result still holds if an arbitrary vector $f_{\varepsilon}^{\prime}=$ $\left(f_{\varepsilon 1}^{\prime}, f_{\varepsilon 2}^{\prime}, 0\right)$ is such that $\left|f_{\varepsilon}^{\prime}\right| \leq \exp \left(-c^{\prime} / \varepsilon\right)$ is added to $f_{\varepsilon}$.

Obviously, $c, c^{\prime}$ and $c_{\alpha}^{\prime \prime}$ depend on the data $g, \nu, l$ and $\eta$. In the sequel, $c, c^{\prime}$ and $c^{\prime \prime}$ will still be used to denote various numbers depending on these data. We will denote by $c_{\alpha}$ or $c_{M}$ numbers that, moreover, depend on $\alpha$ or $M$.

3. Decomposition of the remainder. Let $f$ be an arbitrary vector of $\mathbb{R}^{3}$ such that

$$
f_{3}=0 \text {. }
$$

Replacing in (1) $u_{\varepsilon}$ by the right-hand side of $(3)$ relative to $f_{\varepsilon}=f$, we get the following equations for $\left(w_{\varepsilon}, p_{\varepsilon}\right)$ :

$$
\begin{aligned}
& w_{\varepsilon} \in\left(H_{\mathrm{per}}^{1}\left(\Omega_{\varepsilon}\right)\right)^{3}, \quad p_{\varepsilon} \in L_{\mathrm{per}}^{2}\left(\Omega_{\varepsilon}\right), \\
& -\nu \Delta w_{\varepsilon}+w_{\varepsilon} \cdot \nabla w_{\varepsilon}+w_{\varepsilon 3} \frac{g+\varepsilon f}{l_{3}}+\left(\frac{x_{3}}{l_{3}}(g+\varepsilon f)-\varepsilon f\right) \cdot \nabla w_{\varepsilon}+\nabla p_{\varepsilon}=0, \\
& \nabla \cdot w_{\varepsilon}=0,\left.\quad w_{\varepsilon}\right|_{\mathcal{P}}=0,\left.\quad w_{\varepsilon}\right|_{\mathcal{R}_{\varepsilon}}=-\frac{\eta_{\varepsilon}}{l_{3}}(g+\varepsilon f)+\varepsilon f .
\end{aligned}
$$

We will approximate $w_{\varepsilon}$ by a function $\Psi_{\varepsilon}$ defined in the whole rough half-space $\widetilde{\mathcal{O}}_{\varepsilon}=$ $\left\{x \in \mathbb{R}^{3}: x^{\prime} \in \mathbb{R}^{2}, x_{3}>\eta_{\varepsilon}\left(x^{\prime}\right)\right\}$. This will allow us to use a Saint-Venant type property of exponential decay as $x_{3} \rightarrow \infty$; see (11). The problem on $w_{\varepsilon}$ is close to a problem in such an infinite strip because the aspect ratio length/width of $\Omega_{\varepsilon}$ is $l_{3} /\left(\varepsilon l_{1}\right)$.

To this end, we extend $\Omega_{\varepsilon}$ in the vertical direction by

$$
\widetilde{\Omega}_{\varepsilon}=\left\{x \in \mathbb{R}^{3}: x^{\prime} \in S_{\varepsilon}, x_{3}>\eta_{3}\left(x^{\prime}\right)\right\},
$$


and we consider a corrector $\left(\Psi_{\varepsilon}, \xi_{\varepsilon}\right)$ in the rough half-space $\widetilde{\mathcal{O}}_{\varepsilon}$ that satisfies:

$$
\begin{aligned}
& \Psi_{\varepsilon} \in\left(H_{\text {per.loc }}^{1}\left(\widetilde{\Omega}_{\varepsilon}\right)\right)^{3}, \quad \nabla \Psi_{\varepsilon} \in\left(L_{\mathrm{per}}^{2}\left(\widetilde{\Omega}_{\varepsilon}\right)\right)^{9}, \quad \xi_{\varepsilon} \in L_{\mathrm{per} . \mathrm{loc}}^{2}\left(\widetilde{\Omega}_{\varepsilon}\right) . \\
& -\nu \Delta \Psi_{\varepsilon}+\Psi_{\varepsilon} \cdot \nabla \Psi_{\varepsilon}+\Psi_{\varepsilon 3} \frac{g+\varepsilon f}{l_{3}}+a_{\varepsilon} \cdot \nabla \Psi_{\varepsilon}+\nabla \xi_{\varepsilon}=0, \\
& \nabla \cdot \Psi_{\varepsilon}=0,\left.\quad \Psi_{\varepsilon}\right|_{\mathcal{R}_{\varepsilon}}=-\frac{\eta_{\varepsilon}}{l_{3}}(g+\varepsilon f)+\varepsilon f,
\end{aligned}
$$

where

$$
a_{\varepsilon}\left(x_{3}\right)= \begin{cases}\frac{x_{3}(g+\varepsilon f)}{l_{3}}-\varepsilon f & \text { if } \eta_{\varepsilon} \leq x_{3} \leq l_{3}, \\ \frac{-g x_{3}+g\left(2 l_{3}+\varepsilon \bar{\eta}\right)}{l_{3}+\varepsilon \bar{\eta}} & \text { if } l_{3}<x_{3} \leq 2 l_{3}+\varepsilon \bar{\eta}, \\ 0 & \text { otherwise. }\end{cases}
$$

We use here a function $a_{\varepsilon}$ that vanishes for large enough $x_{3}$ in order to get (11); see (16).

More precisely, we define $\left(\Psi_{\varepsilon}, \xi_{\varepsilon}\right)$ to be the limit as $m \rightarrow \infty$ of the periodic solution $\left(\Psi_{\varepsilon}^{m}, \xi_{\varepsilon}^{m}\right)$ that cancels for $x_{3}=m$, that is, the solution of:

$$
\begin{aligned}
& -\nu \Delta \Psi_{\varepsilon}^{m}+\Psi_{\varepsilon}^{m} \cdot \nabla \Psi_{\varepsilon}^{m}+\Psi_{\varepsilon 3}^{m} \frac{g+\varepsilon f}{l_{3}}+a_{\varepsilon} \cdot \nabla \Psi_{\varepsilon}^{m}+\nabla \xi_{\varepsilon}^{m}=0, \\
& \nabla \cdot \Psi_{\varepsilon}^{m}=0,\left.\quad \Psi_{\varepsilon}^{m}\right|_{\mathcal{R}_{\varepsilon}}=-\frac{\eta_{\varepsilon}}{l_{3}}(g+\varepsilon f)+\varepsilon f,\left.\quad \Psi_{\varepsilon}^{m}\right|_{x_{3}=m}=0 .
\end{aligned}
$$

The approximation $\Psi_{\varepsilon}$ of $w_{\varepsilon}$ does not vanish on $\mathcal{P}$. Thus, we add it to the residue $\left(\Xi_{\varepsilon}, \sigma_{\varepsilon}\right)$ defined in $\mathcal{O}_{\varepsilon}$ by

$$
w_{\varepsilon}=\Psi_{\varepsilon}+\Xi_{\varepsilon}, \quad p_{\varepsilon}=\xi_{\varepsilon}+\sigma_{\varepsilon}
$$

It satisfies

$$
\begin{aligned}
& \Xi_{\varepsilon} \in\left(H_{\mathrm{per}}^{1}\left(\Omega_{\varepsilon}\right)\right)^{3}, \quad \sigma_{\varepsilon} \in L_{\mathrm{per}}^{2}\left(\Omega_{\varepsilon}\right), \\
& \begin{aligned}
&-\nu \Delta \Xi_{\varepsilon}+\Xi_{\varepsilon} \cdot \nabla \Xi_{\varepsilon}+\Xi_{\varepsilon} \cdot \nabla \Psi_{\varepsilon}+\Psi_{\varepsilon} \cdot \nabla \Xi_{\varepsilon}+\Xi_{\varepsilon 3} \frac{g+\varepsilon f}{l_{3}} \\
&+\left(\frac{x_{3}}{l_{3}}(g+\varepsilon f)-\varepsilon f\right) \cdot \nabla \Xi_{\varepsilon}+\nabla \sigma_{\varepsilon}=0, \\
& \nabla \cdot \Xi_{\varepsilon}=0,\left.\quad \Xi_{\varepsilon}\right|_{\mathcal{P}}=-\left.\Psi_{\varepsilon}\right|_{\mathcal{P}},\left.\quad \Xi_{\varepsilon}\right|_{\mathcal{R}_{\varepsilon}}=0 .
\end{aligned}
\end{aligned}
$$

There exist, see Lemma $3, \bar{\Psi}_{\varepsilon \infty} \in \mathbb{R}^{3}$ and $\bar{\xi}_{\varepsilon \infty} \in \mathbb{R}$ and three positive numbers $c, c^{\prime}$ and $c_{\alpha}^{\prime \prime}$ such that, if $\varepsilon<c$, the solution $\left(\Psi_{\varepsilon}, \xi_{\varepsilon}\right)$ of $(6)$ satisfies, for all $x \in \mathcal{O}_{\varepsilon}$ such that $x_{3} \geq \varepsilon(\bar{\eta}+1)$,

$$
\left|\partial^{\alpha}\left(\Psi_{\varepsilon}-\bar{\Psi}_{\varepsilon x}\right)(x)\right|+\left|\partial^{\alpha}\left(\xi_{\varepsilon}-\bar{\xi}_{\varepsilon x}\right)(x)\right| \leq c_{\alpha}^{\prime \prime} \exp \left(-c^{\prime} \frac{x_{3}}{\varepsilon}\right) .
$$

The main point to prove Theorem 1 is to establish that there exists $f$ such that

$$
\bar{\Psi}_{\varepsilon \infty}=0 .
$$

This will be done by proving that, if $\varepsilon$ is small enough, the map $f \mapsto f-\bar{\Psi}_{\varepsilon \infty} / \varepsilon$ is contractive and therefore possesses (at least) one fixed point, which will be denoted $f_{\varepsilon}$. To prove this, we will define $\left(\Phi_{\varepsilon}, \Pi_{\varepsilon}\right)$ by

$$
\Psi_{\varepsilon}=\varepsilon\left(\Phi_{\varepsilon}+f\right), \quad \xi_{\varepsilon}=\varepsilon \Pi_{\varepsilon},
$$


and we will prove (Lemma 8) that the map $f \mapsto-\bar{\Phi}_{\varepsilon \infty}=f-\bar{\Psi}_{\varepsilon \infty} / \varepsilon$ is contractive. The proof will use preliminary estimates on $\Psi_{\varepsilon}$ (Lemma 4), on $\nabla \Phi_{\varepsilon}$ (Lemma 5), on $\overline{\Phi_{\varepsilon}^{2}}-\overline{\Phi_{\varepsilon}^{1}}$ (Lemma 6) and on its gradient (Lemma 7), where $\Phi_{\varepsilon}^{1}$ and $\Phi_{\varepsilon}^{2}$ correspond to distinct data $f^{1}$ and $f^{2}$.

Using (12), we will prove, in Lemma 9, that the $H^{1}$ norm of the residue $\Xi_{\varepsilon}$ goes exponentially fast to 0 as $\varepsilon$ goes to 0 . This will imply the pointwise decay

$$
\left|\partial^{\alpha} \Xi_{\varepsilon}(x)\right|+\left|\partial^{\alpha} \sigma_{\varepsilon}(x)\right| \leq c_{\alpha}^{\prime \prime} \exp \left(\frac{-c^{\prime}}{\varepsilon}\right)
$$

for all $x \in \Omega_{\varepsilon}$ such that $x_{3} \geq \varepsilon(\bar{\eta}+1)$. Together with the pointwise decay (11) and with (12), this will provide the announced decay (4).

4. Saint-Venant estimate. Let $\left(\Psi^{\prime}, \xi^{\prime}\right)$ be a couple of functions in $\mathbb{R}_{+}^{3}$ that are periodic with respect to $x_{i}$ with period $k_{i}, i=1$ or 2 , and that satisfy

$$
\begin{aligned}
& \Psi^{\prime} \in\left(H_{\mathrm{loc}}^{1}\left(\mathbb{R}_{+}^{3}\right)\right)^{3}, \quad \xi^{\prime} \in L_{\mathrm{loc}}^{2}\left(\mathbb{R}_{+}^{3}\right), \quad \int_{\Lambda^{0}}\left|\nabla \Psi^{\prime}\right|^{2} \leq E, \\
& -\nu \Delta \Psi^{\prime}+\Psi^{\prime} \cdot \nabla \Psi^{\prime}+\Psi_{3}^{\prime} b+a \cdot \nabla \Psi^{\prime}+\nabla \xi^{\prime}=0, \\
& \nabla \cdot \Psi^{\prime}=0, \quad \int_{\Sigma} \Psi_{3}^{\prime}\left(x^{\prime}, 0\right) d x^{\prime}=0, \quad\left|\overline{\Psi^{\prime}}(0)\right| \leq E,
\end{aligned}
$$

where $\Sigma=\left(0, k_{1}\right) \times\left(0, k_{2}\right), \Lambda^{t}=\left\{x \in \mathbb{R}^{3}: x^{\prime} \in \Sigma, x_{3}>t\right\}, b \in \mathbb{R}^{3}, b_{3}=0$ and

$$
a=a\left(x_{3}\right), \quad a_{3}=0, \quad a=0 \text { in }\left[2 l_{3},+\infty\right), \quad a \in\left(W^{1, \infty}(0, \infty)\right)^{3} .
$$

LEMmA 3. There exists $\bar{\Psi}_{\infty}^{\prime} \in \mathbb{R}^{3}$ such that $\bar{\Psi}_{\infty 3}^{\prime}=0$ and

$$
\left|\bar{\Psi}^{\prime}(t)-\bar{\Psi}_{\infty}^{\prime}\right| \leq \frac{\kappa K^{2}}{\nu k_{1} k_{2}} \int_{\Lambda^{t}}\left|\nabla \Psi^{\prime}\right|^{2}
$$

where $\kappa$ is a universal constant and $K=\max \left(k_{1}, k_{2}\right)$.

If $K \leq \frac{1}{2 \kappa} \sqrt{\nu /|b|}$, one has, for all $t \geq K$,

$$
\left\|\nabla \Psi^{\prime}\right\|_{\left(L^{2}\left(\Lambda^{t}\right)\right)^{9}} \leq \mu\left(\int_{\Lambda^{0}}\left|\nabla \Psi^{\prime}\right|^{2}\right)^{1 / 2} \exp \left(-\frac{\lambda t}{K}\right)
$$

where $\lambda>0$. For all $\alpha \in \mathbb{N}^{3}$ and $t \geq K$, one has

$$
\left\|\Psi^{\prime}-\Psi_{\infty}^{\prime}\right\|_{\left(W^{\alpha, \infty}\left(\Lambda^{t}\right)\right)^{3}}+\left\|\xi^{\prime}\right\|_{W^{\alpha, \infty}\left(\Lambda^{t}\right)} \leq \sigma_{\alpha} K^{-(m+2)}\left\|\Psi^{\prime}-\Psi_{\infty}^{\prime}\right\|_{\left(H^{1}\left(\Lambda^{t-K}\right)\right)^{3}} .
$$

Given a positive real number $c$, one can choose $\lambda, \mu$ and $\sigma_{\alpha}$ such that (18) and (19) hold for all $a, b, k_{1}$ and $k_{2}$ such that $|a| \leq c,|b| \leq c, k_{1} / k_{2} \leq c$ and $k_{2} / k_{1} \leq c$.

For the reader's convenience, let us give hints about the proof of Lemma 3, which is rather complicated. In the much simpler case of Laplace's equation, a proof based on an explicit decomposition on a special basis is given in [5], Lemma 4 . In the case of stationary Navier-Stokes equations in a semi-infinite channel with lateral Dirichlet boundary condition, a similar result is proved in [10], Vol. 2, Theorem 4.1, p. 261; see also references therein.

Here, the lateral Dirichlet boundary condition is replaced by a periodicity condition, and therefore the mean value $\bar{\Psi}^{\prime}(t)=(1 /|\Sigma|) \int_{\Sigma} \Psi^{\prime}\left(x^{\prime}, t^{\prime}\right) d x^{\prime}$ may no longer be bounded 
by using the Poincaré inequality as in [10]. However, a similar method may be used, using the Poincaré-Wirtinger inequality and the fact that $\bar{\Psi}^{\prime}(t)$ remains bounded. This is done in [4], Theorem 2.

Here, $\Psi^{\prime}$ is not governed by stationary Navier-Stokes equations; it is governed by (15), which contains the extra term $\Psi_{3}^{\prime} b+a \cdot \nabla \Psi^{\prime}$. However, the proof of [4] extends to this case, the extra term being bounded by similar calculus; this is done in [3], Theorem 2 and its proof. Outlines are as follows.

In a first step, using the fact that $\nabla \Psi^{\prime} \in\left(L^{2}\left(\Lambda^{0}\right)\right)^{9}$, we prove that, for $i=1$ or 2 ,

$$
\frac{d \bar{\Psi}_{i}^{\prime}}{d t}=\frac{1}{\nu k_{1} k_{2}} \int_{\Sigma}\left(\Psi_{3}^{\prime} \Psi_{i}^{\prime}\right)\left(x^{\prime}, t\right) d x^{\prime} .
$$

It follows that $\bar{\Psi}^{\prime}(t)$ goes to a limit $\bar{\Psi}_{\infty}^{\prime}$ as $t \rightarrow \infty$ and that (17) is satisfied.

In a second step, we prove that, if $K \leq \sqrt{\nu /|b|} /(2 \kappa)$, then $y(t)=\int_{\Lambda^{t}}\left|\nabla \Psi^{\prime}\right|^{2} d x^{\prime}$ satisfies the following ordinary differential inequality, with $c_{i}$ independent of $K$ :

$$
\frac{d y}{d t}(t)+\frac{c_{1}}{K} \int_{t}^{\infty} y(\tau) d \tau \leq \frac{c_{2}}{K} y(t)
$$

This inequality implies $|y(t)| \leq c_{3} y(0) \exp \left(-c_{4} t / K\right)$ and thus (18).

Finally, the pointwise decay (19) follows from regularity properties of solutions of Stokes equations, as in [4], proof of Theorem 2, pp. 363-364.

5. Properties of $\Psi_{\varepsilon}$. Let us define, for $t \geq \varepsilon \bar{\eta}$,

$$
\bar{\Psi}_{\varepsilon}(t)=\frac{1}{l_{1} l_{2}} \int_{S} \Psi_{\varepsilon}\left(x^{\prime}, t\right) d x^{\prime} .
$$

Remark that (11) implies that

$$
\bar{\Psi}_{\varepsilon \infty}=\lim _{t \rightarrow+\infty} \bar{\Psi}_{\varepsilon}(t)
$$

Lemma 4. Given $M>0$, there exists a positive number $c_{M}$ such that, if $|f| \leq M$ and $\varepsilon<c_{M I}$, then

$$
\begin{gathered}
\int_{\tilde{\Omega}_{\varepsilon}}\left|\nabla \Psi_{\varepsilon}\right|^{2} \leq c \varepsilon^{3}(|g|+\varepsilon M), \\
\left|\bar{\Psi}_{\varepsilon}(\varepsilon \bar{\eta})\right| \leq c \varepsilon(|g|+\varepsilon M),
\end{gathered}
$$

where $c$ does not depend on $M$. For all $t \geq \varepsilon \bar{\eta}$,

$$
\begin{gathered}
\bar{\Psi}_{\varepsilon 3}(t)=0, \\
\left|\bar{\Psi}_{\varepsilon}(t)-\bar{\Psi}_{\varepsilon \infty}\right| \leq \frac{\kappa c_{l}}{\nu} \int_{\Theta^{t}}\left|\nabla \Psi_{\varepsilon}\right|^{2},
\end{gathered}
$$

where $c_{l}=\max \left(l_{1} / l_{2}, l_{2} / l_{1}\right)$.

We denote by $\Theta^{t}$ the semi-infinite vertical strip defined by

$$
\Theta^{t}=\left\{x \in \mathbb{R}^{3}: x^{\prime} \in S_{\varepsilon}, x_{3}>t\right\} .
$$

The dependence on $\varepsilon$ of $\Theta^{t}$ and of other quantities defined later on is not pointed out by a subscript in order to reduce notation. 
Proof of (22). Let $\Psi_{\varepsilon}^{m}$ be the approximation of $\Psi_{\varepsilon}$ defined by (8). Let us first prove that, for all $t \geq \varepsilon \bar{\eta}$,

$$
\bar{\Psi}_{\varepsilon 3}^{m}(t)=0 .
$$

If $m \leq t$, it is obvious since $\Psi_{\varepsilon}^{m}$ cancels if $x_{3} \geq m$. Else, let $\Lambda=\left\{x: x^{\prime} \in S_{\varepsilon}, t<x_{3}<m\right\}$ and $\Sigma^{t}=\left\{x: x^{\prime} \in S, x_{3}=t\right\}$. The incompressibility condition gives

$$
0=\int_{\Lambda} \nabla \cdot \Psi_{\varepsilon}=\int_{\Sigma^{t} \cup \Sigma^{m} \cup L} \Psi_{\varepsilon} \cdot n d s
$$

where $L$ is the lateral part of the boundary of $\Lambda$ and $n$ is the unit outward vector field. The integral over $L$ cancels since $\Psi_{\varepsilon}$ is periodic with respect to $x_{1}$ and $x_{2}$, and the integral over $S^{m}$ cancels since $\Psi_{\varepsilon}^{m}$ vanishes on it. Then,

$$
\int_{S^{t}} \Psi_{\varepsilon 3}^{m} d s=0
$$

This gives (24), and (22) follows by passing to the limit as $m \rightarrow \infty$.

Proof of (20). Let $\Psi_{\varepsilon}^{m}$ be again the approximation of $\Psi_{\varepsilon}$ defined by (8). Let us consider the extension in $\widetilde{\mathcal{O}}_{\varepsilon}$ of the trace of $\Psi_{\varepsilon}$ on $\mathcal{R}_{\varepsilon}$ given by

$$
h\left(x^{\prime}, x_{3}\right)= \begin{cases}-\frac{x_{3}}{l_{3}}(g+\varepsilon f)+\varepsilon f & \text { if } \eta_{\varepsilon} \leq x_{3}<\varepsilon \bar{\eta}, \\ \left(-\frac{\varepsilon \bar{\eta}}{l_{3}}(g+\varepsilon f)+\varepsilon f\right) \frac{2 x_{3}-l_{3}}{2 \varepsilon \bar{\eta}-l_{3}} & \text { if } \varepsilon \bar{\eta} \leq x_{3}<l_{3} / 2, \\ 0 & \text { otherwise. }\end{cases}
$$

Let $\psi=\Psi_{\varepsilon}^{m}-h$ and $\xi=\xi_{\varepsilon}^{m}$ where $m \geq l_{3} / 2$, and let $\Omega_{\varepsilon}^{m}=\left\{x \in \widetilde{\Omega}_{\varepsilon}: x_{3}<m\right\}$. In $\Omega_{\varepsilon}^{m}$, the pair $(\psi, \xi)$ satisfies

$$
\begin{aligned}
& -\nu \Delta \psi-\nu \Delta h+\psi \cdot \nabla \psi+h \cdot \nabla \psi+\psi \cdot \nabla h+\psi_{3} \frac{g+\varepsilon f}{l_{3}}+a_{\varepsilon} \cdot \nabla \psi+\nabla \xi=0, \\
& \nabla \cdot \psi=0,\left.\quad \psi\right|_{\mathcal{R}_{\varepsilon}}=0,\left.\quad \psi\right|_{x_{3}=m}=0 .
\end{aligned}
$$

Multiplying the first equation in (26) by $\psi$ and integrating by parts over $\Omega_{\varepsilon}^{m}$, we obtain

$$
\nu \int_{\Omega_{\varepsilon}^{m}}|\nabla \psi|^{2}+\nu \int_{\Omega_{\varepsilon}^{m}} \nabla \psi \cdot \nabla h+\int_{\Omega_{\varepsilon}^{m}} \psi \cdot \nabla h \cdot \psi+\int_{\Omega_{\varepsilon}^{m}} \psi_{3} \frac{g+\varepsilon f}{l_{3}} \cdot \psi=0 .
$$

Let us estimate the last three integrals. Using the definition of $h$ and (24), we get

$$
\begin{aligned}
\left|\int_{\Omega_{\varepsilon}^{m}} \nabla \psi \cdot \nabla h\right| & \leq \frac{1}{2} \int_{\Omega_{\varepsilon}^{m}}|\nabla \psi|^{2}+c \varepsilon^{3}(|g|+\varepsilon M), \\
\left|\int_{\Omega_{\varepsilon}^{m}} \psi \cdot \nabla h \cdot \psi_{3}\right| & \leq c \varepsilon^{2}(|g|+M) \int_{\Omega_{\varepsilon}^{m}}|\nabla \psi|^{2},
\end{aligned}
$$

and

$$
\left|\int_{\Omega_{\varepsilon}^{m}} \psi_{3} \frac{g+\varepsilon f}{l_{3}} \cdot \psi\right| \leq c \varepsilon^{2}(|g|+M) \int_{\Omega_{\varepsilon}^{m}}|\nabla \psi|^{2} .
$$

Inequality (20) comes from (27) with these three estimates by using the decomposition $\Psi_{\varepsilon}^{m}=\psi+h$ and by passing to the limit over $m$. 
Proof of (21). Let us notice that

$$
\Psi_{\varepsilon}\left(x^{\prime}, \varepsilon \bar{\eta}\right)=\int_{\eta_{\varepsilon}\left(x^{\prime}\right)}^{\varepsilon \bar{\eta}} \partial_{z} \Psi_{\varepsilon}\left(x^{\prime}, z\right) d z-\frac{\eta_{\varepsilon}\left(x^{\prime}\right)}{l_{3}}(g+\varepsilon f)+\varepsilon f .
$$

Integrating with respect to $x^{\prime}$, we get

$$
\left|\bar{\Psi}_{\varepsilon}(\varepsilon \bar{\eta})\right| \leq \frac{1}{\varepsilon^{2} l_{1} l_{2}} \int_{S_{\varepsilon}} \int_{\eta_{\varepsilon}\left(x^{\prime}\right)}^{\varepsilon \bar{\eta}}\left|\partial_{z} \Psi_{\varepsilon}\left(x^{\prime}, z\right)\right| d z d x^{\prime}+\varepsilon\left(1+\frac{\bar{\eta}}{l_{3}}\right)(|g|+\varepsilon M I) .
$$

Using the Cauchy-Schwarz inequality and (20), we get (21).

Proof of (23). Thanks to Lemma 3, (23) follows from the momentum equation in (21), from (5) and from (22), since $\nabla \Psi_{\varepsilon} \in\left(L^{2}\left(\Theta^{t}\right)\right)^{9}$ by $(20)$.

6. Exponential decay of $\nabla \Phi_{\varepsilon}$. Notice that, by (13), $\nabla \Phi_{\varepsilon}=\nabla \Psi_{\varepsilon} / \varepsilon$.

Lemma 5. Given $M>0$, there exists a positive number (' $M$ such that, if $|f| \leq M$, $\varepsilon<c_{M}$ and $x_{3} \geq \varepsilon(\bar{\eta}+1)$, then

$$
\left|\nabla \Phi_{\varepsilon}\left(x^{\prime} . x_{3}\right)\right| \leq c_{M I}^{\prime} \sqrt{\varepsilon} \exp \left(-c_{M}^{\prime \prime} \frac{x_{3}}{\varepsilon}\right) .
$$

Proof. Let us shift $\left(\Psi_{\varepsilon}, \xi_{\varepsilon}\right)$ by $\varepsilon \bar{\eta}$ in the vertical direction, that is, let

$$
\left(\Psi^{\prime}, \xi^{\prime}\right)\left(x^{\prime}, x_{3}\right)=\left(\Psi_{\varepsilon}, \xi_{\varepsilon}\right)\left(x^{\prime}, x_{3}+\varepsilon \bar{\eta}\right)
$$

for all $\left(x^{\prime}, x_{3}\right) \in \mathbb{R}_{+}^{3}$. By (6), (7), (20), and (21), the pair $\left(\Psi^{\prime}, \xi^{\prime}\right)$ satisfies (15) with $k_{1}=\varepsilon l_{1}, k_{2}=\varepsilon l_{2}$ and $E=\varepsilon^{3} c_{M I}^{\prime \prime \prime}\left(|g|+c_{\Lambda} M\right)$. Then Lemma 3 gives

$$
\left|\nabla \Psi^{\prime}\left(x^{\prime} . x_{3}\right)\right| \leq c_{M I}^{\prime} \varepsilon^{3 / 2} \exp \left(-c_{M}^{\prime \prime} \frac{x_{3}}{\varepsilon}\right)
$$

for all $x_{3} \geq \varepsilon$. This implies (28) since, for all $x_{3} \geq \varepsilon(\bar{\eta}+1)$,

$$
\nabla \Phi_{\varepsilon}\left(x^{\prime}, x_{3}\right)=\frac{1}{\varepsilon} \nabla \Psi^{\prime}\left(x^{\prime}, x_{3}-\varepsilon \bar{\eta}\right) .
$$

7. Estimate of $\Phi_{\varepsilon}^{2}-\Phi_{\varepsilon}^{1}$. Let $\left(\Psi_{\varepsilon}^{1}, \xi_{\varepsilon}^{1}\right)$ and $\left(\Psi_{\varepsilon}^{2}, \xi_{\varepsilon}^{2}\right)$ be the solutions of $(6)$ associated, respectively, with two different vectors $f^{1}$ and $f^{2}$ such that $f_{3}^{1}=f_{3}^{2}=0$. More precisely, they are the limits as $m \rightarrow \infty$ of the corresponding approximate solutions $\Psi_{\varepsilon}^{m 1}$ and $\Psi_{\varepsilon}^{m 2}$ of (8). We denote

$$
\Phi_{\varepsilon}^{1}=\frac{\Psi_{\varepsilon}^{1}}{\varepsilon}-f^{1}, \quad \Phi_{\varepsilon}^{2}=\frac{\Psi_{\varepsilon}^{2}}{\varepsilon}-f^{2} .
$$

Lemma 6. If $\left|f^{1}\right| \leq M$ and $\left|f^{2}\right| \leq M$, then, for any $t>\varepsilon \bar{\eta}$,

$$
\left|\left(\overline{\Phi_{\varepsilon}^{2}}-\overline{\Phi_{\varepsilon}^{1}}\right)(t)\right| \leq c_{\Lambda}\left(\left(1+\frac{1}{\sqrt{\varepsilon}}\right)\left(\int_{\tilde{\Omega}_{\varepsilon}}\left|\nabla\left(\Phi_{\varepsilon}^{2}-\Phi_{\varepsilon}^{1}\right)\right|^{2}\right)^{1 / 2}+\varepsilon\left|f^{2}-f^{1}\right|\right) .
$$

Proof. Let us denote

$$
f=f^{1}-f^{2} . \quad \Phi=\Phi_{\varepsilon}^{1}-\Phi_{\varepsilon}^{2}, \quad \pi=\pi_{\varepsilon}^{1}-\pi_{\varepsilon}^{2} .
$$


From $(6),(\Phi, \pi)$ satisfies

$$
\begin{aligned}
& \Phi \in\left(H_{\text {per }, \text { loc }}^{1}\left(\widetilde{\Omega}_{\varepsilon}\right)\right)^{3}, \quad \pi \in L_{\text {per }, \text { loc }}^{2}\left(\widetilde{\Omega}_{\varepsilon}\right), \quad \int_{\tilde{\Omega}_{\varepsilon}}|\nabla \Phi|^{2}<\infty, \\
& -\nu \Delta \Phi+\varepsilon \Phi_{\varepsilon}^{1} \cdot \nabla \Phi+\varepsilon \Phi \cdot \nabla \Phi_{\varepsilon}^{2}+\varepsilon \Phi_{3}^{1} \frac{f}{l_{3}} \\
& \quad+\Phi_{3} \frac{g+\varepsilon f^{2}}{l_{3}}+\left(a_{\varepsilon}^{1}+\varepsilon f^{1}\right) \cdot \nabla \Phi+\left(a_{\varepsilon}^{1}-a_{\varepsilon}^{2}+\varepsilon f\right) \cdot \nabla \Phi_{\varepsilon}^{2}+\nabla \pi=0, \\
& \nabla \cdot \Phi=0,\left.\quad \Phi\right|_{\mathcal{R}_{\varepsilon}}=-\frac{\eta_{\varepsilon} f}{l_{3}} .
\end{aligned}
$$

Obviously,

$$
|\bar{\Phi}(t)| \leq|\bar{\Phi}(t)-\bar{\Phi}(\varepsilon(\bar{\eta}+1))|+|\bar{\Phi}(\varepsilon(\bar{\eta}+1))| .
$$

In a similar way to (23), integrating on $S^{\varepsilon}$ the horizontal momentum equations given in $(30)$, for $t \geq \varepsilon(\bar{\eta}+1)$ fixed, and using the periodicity of the solution, we get

$$
|\bar{\Phi}(t)-\bar{\Phi}(\varepsilon(\bar{\eta}+1))| \leq \frac{c}{\varepsilon}\left(\left\|\nabla \Phi_{\varepsilon}^{1}\right\|_{\left(L^{2}\left(\Theta^{\varepsilon(\bar{\eta}+1)}\right)\right)^{9}}+\left\|\nabla \Phi_{\varepsilon}^{2}\right\|_{\left(L^{2}\left(\Theta^{\varepsilon(\bar{\eta}+1)}\right)\right)^{9}}\right)\|\nabla \Phi\|_{\left(L^{2}\left(\Theta^{\varepsilon(\bar{\eta}+1)}\right)\right)^{9}} .
$$

Therefore, by (28),

$$
|\bar{\Phi}(t)-\bar{\Phi}(\varepsilon(\bar{\eta}+1))| \leq c_{M}\|\nabla \Phi\|_{\left(L^{2}\left(\Theta^{\varepsilon(\bar{\eta}+1)}\right)\right)^{9}} .
$$

On the other hand,

$$
\Phi\left(x^{\prime}, z\right)=\int_{\eta_{\varepsilon}\left(x^{\prime}\right)}^{z} \partial_{z} \Phi\left(x^{\prime}, \zeta\right) d \zeta-\frac{\eta_{\varepsilon}\left(x^{\prime}\right)}{l_{3}} f .
$$

Therefore, choosing $z=\varepsilon(\bar{\eta}+1)$ and integrating with respect to $x^{\prime}$, we get

$$
|\bar{\Phi}(\varepsilon(\bar{\eta}+1))| \leq \frac{c}{\sqrt{\varepsilon}}\left\|\partial_{z} \Phi\right\|_{\left(L^{2}\left(\tilde{\Omega}_{\varepsilon}\right)\right)^{3}}+c \varepsilon|f| .
$$

Using (31), (32), and (34), we get (29).

In order to bound the right-hand side of (29) in terms of $c\left|f^{2}-f^{1}\right|$, let us prove the following result.

Lemma 7. There exist positive numbers $c_{\Lambda I}$ and $c_{M I}^{\prime}$ such that, if $\left|f^{1}\right| \leq M$ and $\left|f^{2}\right| \leq M$ and $\varepsilon<c_{M}$,

$$
\left\|\nabla\left(\Phi_{\varepsilon}^{2}-\Phi_{\varepsilon}^{1}\right)\right\|_{\left(L^{2}\left(\tilde{\Omega}_{\varepsilon}\right)\right)^{9}} \leq c_{\Lambda I}^{\prime} \varepsilon^{3 / 2}\left|f^{2}-f^{1}\right| .
$$

Proof. In order to get a homogeneous boundary condition on $\mathcal{R}_{\varepsilon}$, let $k$ be the extension of the trace of $\Phi$ on $\mathcal{R}_{\varepsilon}$ defined by

$$
k\left(x^{\prime}, x_{3}\right)= \begin{cases}-\frac{x_{3}}{l_{3}} f & \text { if } \eta_{\varepsilon} \leq x_{3}<\varepsilon \bar{\eta} \\ -\varepsilon \frac{\bar{\eta} f\left(2 x_{3}-l_{3}\right)}{l_{3}\left(2 \varepsilon \bar{\eta}-l_{3}\right)} & \text { if } \varepsilon \bar{\eta} \leq x_{3}<l_{3} / 2 \\ 0 & \text { otherwise. }\end{cases}
$$


Let us set $\phi=\Phi-k$. Then $(\phi, \pi)$ satisfies

$$
\begin{aligned}
& \phi \in\left(H_{\mathrm{per}, \mathrm{loc}}^{1}\left(\widetilde{\Omega}_{\varepsilon}\right)\right)^{3}, \quad \pi \in L_{\mathrm{per}, \mathrm{loc}}^{2}\left(\widetilde{\Omega}_{\varepsilon}\right), \quad \int_{\widetilde{\Omega}_{\varepsilon}}|\nabla \phi|^{2}<\infty, \\
& -\nu \Delta \phi+\varepsilon \Phi_{\varepsilon}^{1} \cdot \nabla \phi+\varepsilon \phi \cdot \nabla \Phi_{\varepsilon}^{2}+\varepsilon \Phi_{\varepsilon 3}^{1} \frac{f}{l_{3}}+\phi_{3} \frac{g+\varepsilon f^{2}}{l_{3}}+\left(a_{\varepsilon}^{1}+\varepsilon f^{1}\right) \cdot \nabla \phi \\
& \quad+\left(a_{\varepsilon}^{1}-a_{\varepsilon}^{2}+\varepsilon f\right) \cdot \nabla \Phi_{\varepsilon}^{2}-\nu \Delta k+\varepsilon \Phi_{\varepsilon}^{1} \cdot \nabla k+\varepsilon k \cdot \nabla \Phi_{\varepsilon}^{2}+\nabla \pi=0, \\
& \nabla \cdot \phi=0,\left.\quad \phi\right|_{\mathcal{R}_{\varepsilon}}=0,
\end{aligned}
$$

where $a_{\varepsilon}^{1}$ and $a_{\varepsilon}^{2}$ are the functions associated to $f^{1}$ and $f^{2}$ by (7). Multiplying by $\phi$ the momentum equation in (36), we get

$$
\begin{aligned}
\nu \int_{\widetilde{\Omega}_{\varepsilon}}|\nabla \phi|^{2}= & -\varepsilon \int_{\widetilde{\Omega}_{\varepsilon}} \phi \cdot \nabla \Phi_{\varepsilon}^{2} \cdot \phi-\varepsilon \int_{\widetilde{\Omega}_{\varepsilon}} \Phi_{\varepsilon 3}^{1} \frac{f}{l_{3}} \cdot \phi-\int_{\widetilde{\Omega}_{\varepsilon}} \phi_{3} \frac{g+\varepsilon f^{2}}{l_{3}} \cdot \phi \\
& -\int_{\widetilde{\Omega}_{\varepsilon}}\left(a_{\varepsilon}^{1}-a_{\varepsilon}^{2}+\varepsilon f\right) \cdot \nabla \Phi_{\varepsilon}^{2} \cdot \phi-\nu \int_{\widetilde{\Omega}_{\varepsilon}} \nabla k \cdot \nabla \phi \\
& -\varepsilon \int_{\widetilde{\Omega}_{\varepsilon}} \Phi_{\varepsilon}^{1} \cdot \nabla k \cdot \phi-\varepsilon \int_{\widetilde{\Omega}_{\varepsilon}} k \cdot \nabla \Phi_{\varepsilon}^{2} \cdot \phi \\
= & I_{1}+\cdots+I_{7} .
\end{aligned}
$$

This integration by parts may be justified by passing to the limit on the approximate solutions $\Psi_{m}$. It may also be justified by remarking that $-\nu \Delta \phi+\nabla \pi=\nabla \cdot(-\nu \nabla \phi+\pi$ Id $)$, which is in $L^{1}$ and which is in $L^{3 / 2}$ in any bounded set thanks to (28), (36) and the fact that the $\Phi_{\varepsilon}^{i}$ are bounded outside a neighbourhood of the boundary.

Let us estimate $I_{1}, I_{2}, \ldots, I_{7}$ in terms of $\int_{\widetilde{\Omega}_{\varepsilon}}|\nabla \phi|^{2}$. We will decompose the integral over $\widetilde{\Omega}_{\varepsilon}$ into an integral over the infinite strip with flat bottom

$$
\Theta^{\prime}=\left\{x \in \mathbb{R}^{3}: x^{\prime} \in S_{\varepsilon}, \quad x_{3}>2 l_{3}+\varepsilon \bar{\eta}\right\}
$$

and an integral over the bounded domain

$$
\Lambda^{\prime}=\left\{x \in \mathbb{R}^{3}: x^{\prime} \in S_{\varepsilon}, \quad \eta_{\varepsilon}\left(x^{\prime}\right)<x_{3}<2 l_{3}+2 \varepsilon \bar{\eta}\right\} .
$$

We will use the Poincaré-Wirtinger inequality in $\Theta^{\prime}$, and the Poincaré inequality in $\Lambda^{\prime}$.

Since $\int_{\Theta^{\prime}} \bar{\phi} \cdot \nabla \Phi_{\varepsilon}^{2} \cdot \bar{\phi}=0$, we can write

$$
\begin{array}{r}
I_{1}=-\varepsilon \int_{\Theta^{\prime}}(\phi-\bar{\phi}) \cdot \nabla \Phi_{\varepsilon}^{2} \cdot(\phi-\bar{\phi})+\bar{\phi} \cdot \nabla \Phi_{\varepsilon}^{2} \cdot(\phi-\bar{\phi})+(\phi-\bar{\phi}) \cdot \nabla \Phi_{\varepsilon}^{2} \cdot \bar{\phi} \\
-\varepsilon \int_{\Lambda^{\prime}} \phi \cdot \nabla \Phi_{\varepsilon}^{2} \cdot \phi
\end{array}
$$

Using the Hölder inequality it follows that

$$
\begin{aligned}
& \left|I_{1}\right| \leq \varepsilon\left\|\nabla \Phi_{\varepsilon}^{2}\right\|_{\left(L^{\infty}\left(\Theta^{\prime}\right)\right)^{9}}\left(\|\phi-\bar{\phi}\|_{\left(L^{2}\left(\Theta^{\prime}\right)\right)^{3}}\right)^{2} \\
& +2 \varepsilon\|\bar{\phi}\|_{\left(L^{\infty}\left(\Theta^{\prime}\right)\right)^{3}}\left\|\nabla \Phi_{\varepsilon}^{2}\right\|_{\left(L^{2}\left(\Theta^{\prime}\right)\right)^{9}}\left(\|\phi-\bar{\phi}\|_{\left.\left(L^{2}\left(\Theta^{\prime}\right)\right)^{3}\right)^{2}}\right. \\
& +c \varepsilon^{4 / 3}\left(\|\phi\|_{\left.\left(L^{6}\left(\Lambda^{\prime}\right)\right)^{3}\right)^{2}}\left\|\nabla \Phi_{\varepsilon}^{2}\right\|_{\left(L^{2}\left(\Lambda^{\prime}\right)\right)^{9}} .\right.
\end{aligned}
$$

From (28) we have

$$
\left\|\nabla \Phi_{\varepsilon}^{2}\right\|_{\left(L^{\infty}\left(\Theta^{\prime}\right)\right)^{9}} \leq c_{M} \sqrt{\varepsilon}
$$


and from the Poincaré-Wirtinger inequality,

$$
\|\phi-\bar{\phi}\|_{\left(L^{2}\left(\Theta^{\prime}\right)\right)^{3}} \leq c \varepsilon\left\|\nabla_{x^{\prime}} \phi\right\|_{\left(L^{2}\left(\Theta^{\prime}\right)\right)^{6}} .
$$

Thanks to $(28)$,

$$
\left\|\nabla \Phi_{\varepsilon}^{2}\right\|_{\left(L^{2}\left(\Theta^{\prime}\right)\right)^{9}} \leq c_{M} \varepsilon^{3 / 2} .
$$

Since $k=0$ in $\Theta^{\prime},(29)$ and (34) give

$$
\|\bar{\phi}\|_{\left(L^{\infty}\left(\Theta^{\prime}\right)\right)^{3}} \leq \frac{c_{M}}{\sqrt{\varepsilon}}\|\nabla \phi\|_{\left(L^{2}\left(\Theta^{\prime}\right)\right)^{9}}+\varepsilon|f| .
$$

Using the change of variable $x^{\prime} \mapsto x^{\prime} / \varepsilon$, which maps the domain $\Lambda^{\prime}$ onto a domain of section $\left(0, l_{1}\right) \times\left(0, l_{2}\right)$, and the fact that $\left.\phi\right|_{\mathcal{R}_{\varepsilon}}=0$, we get

$$
\|\phi\|_{\left(L^{6}\left(\Lambda^{\prime}\right)\right)^{3}} \leq c \varepsilon^{-2 / 3}\|\nabla \phi\|_{\left(L^{2}\left(\Lambda^{\prime}\right)\right)^{9}} .
$$

Using (39), (40), (41), (42), and (43), the inequality (38) gives

$$
\left|I_{1}\right| \leq c_{M} \varepsilon^{1 / 2}\|\nabla \phi\|_{\left(L^{2}\left(\tilde{\Omega}_{\varepsilon}\right)\right)^{9}}\left(\|\nabla \phi\|_{\left(L^{2}\left(\tilde{\Omega}_{\varepsilon}\right)\right)^{9}}+\varepsilon^{3}|f|\right) .
$$

Let us now bound $I_{2}$. There we decompose the integral over $\widetilde{\Omega}_{\varepsilon}$ into integrals over

$$
\begin{gathered}
\Theta^{\prime \prime}=\left\{x \in \mathbb{R}^{3}: x^{\prime} \in S_{\varepsilon}, \quad x_{3}>\varepsilon \bar{\eta}\right\}, \\
\Lambda^{\prime \prime}=\left\{x \in \mathbb{R}^{3}: x^{\prime} \in S_{\varepsilon}, \quad \eta_{\varepsilon}\left(x^{\prime}\right)<x_{3}<\varepsilon \bar{\eta}\right\} .
\end{gathered}
$$

We use a domain $\Lambda^{\prime \prime}$ of vertical size $c \varepsilon$ to get the coefficient $\varepsilon$ in (45). Using (22),

$$
I_{2}=-\varepsilon \int_{\Theta^{\prime \prime}}\left(\Phi_{\varepsilon 3}^{1}-\bar{\Phi}_{\varepsilon 3}^{1}\right) \frac{f}{l_{3}}(\phi-\bar{\phi})-\varepsilon \int_{\Lambda^{\prime \prime}} \Phi_{3 \varepsilon}^{1} \frac{f}{l_{3}} \phi .
$$

We bound the first integral using (40) for $\phi$ and $\Phi^{1}$ (with $\Theta^{\prime \prime}$ instead of $\Theta^{\prime}$ ), (13) and (20). To bound the second integral, we use the following Poincaré inequality:

$$
\|\phi\|_{\left(L^{2}\left(\Lambda^{\prime \prime}\right)\right)^{3}} \leq c \varepsilon\|\nabla \phi\|_{\left(L^{2}\left(\Lambda^{\prime \prime}\right)\right)^{9}}
$$

and

$$
\left\|\Phi_{\varepsilon 3}^{1}\right\|_{\left(L^{2}\left(\Lambda^{\prime \prime}\right)\right)^{3}} \leq c_{M} \varepsilon^{3 / 2} .
$$

This last inequality comes from (33) by using (13) and (20). Thus, we get

$$
\left|I_{2}\right| \leq c_{M} \varepsilon^{7 / 2}|f|\|\nabla \phi\|_{\left(L^{2}\left(\tilde{\Omega}_{\varepsilon}\right)\right)^{9}} .
$$

Similarly, we have

$$
\left|I_{3}\right| \leq c_{M} \varepsilon^{7 / 2}\left(\|\nabla \phi\|_{\left(L^{2}\left(\tilde{\Omega}_{\varepsilon}\right)\right)^{9}}\right)^{2} .
$$

Since $a_{\varepsilon}^{1}=a_{\varepsilon}^{2}=0$ on $\left[2 l_{3}+\varepsilon \bar{\eta},+\infty\right)$, we can write

$$
I_{4}=-\int_{\Theta^{\prime}}\left(\varepsilon f \cdot \nabla \Phi_{\varepsilon}^{2} \cdot(\phi-\bar{\phi})+\varepsilon f \cdot \nabla \Phi_{\varepsilon}^{2} \cdot \bar{\phi}\right)-\int_{\Lambda^{\prime}}\left(a_{\varepsilon}^{1}-a_{\varepsilon}^{2}+\varepsilon f\right) \cdot \nabla \Phi_{\varepsilon}^{2} \cdot \phi .
$$

It follows that

$$
\begin{aligned}
\left|I_{4}\right| \leq & \varepsilon|f|\left(\|\phi-\bar{\phi}\|_{\left(L^{2}\left(\Theta^{\prime}\right)\right)^{3}}\left\|\nabla \Phi_{\varepsilon}^{2}\right\|_{\left(L^{2}\left(\Theta^{\prime}\right)\right)^{9}}+\left\|\nabla \Phi_{\varepsilon}^{2}\right\|_{\left(L^{1}\left(\Theta^{\prime}\right)\right)^{9}}\|\bar{\phi}\|_{\left.\left(L^{\infty}\left(\Theta^{\prime}\right)\right)^{3}\right)}\right. \\
& +\left(\varepsilon|f|+\left\|a_{\varepsilon}^{1}-a_{\varepsilon}^{2}\right\|_{\left(L^{\infty}\left(\Lambda^{\prime}\right)\right)^{3}}\right)\left\|\nabla \Phi_{\varepsilon}^{2}\right\|_{\left(L^{2}\left(\Lambda^{\prime}\right)\right)^{9}}\|\phi\|_{\left(L^{2}\left(\Lambda^{\prime}\right)\right)^{3}} .
\end{aligned}
$$


Thanks to $(28)$,

$$
\left\|\nabla \Phi_{\varepsilon}^{2}\right\|_{\left(L^{1}\left(\Theta^{\prime}\right)\right)^{9}} \leq c_{M} \varepsilon^{3 / 2} .
$$

Then using (40), (41), (42), (48) and

$$
\left\|a_{\varepsilon}^{1}-a_{\varepsilon}^{2}\right\|_{\left(L^{\infty}\left(\Lambda^{\prime}\right)\right)^{3}} \leq c \varepsilon|f|
$$

and using the Poincaré inequality

$$
\|\phi\|_{\left(L^{2}\left(\Lambda^{\prime}\right)\right)^{3}} \leq c\|\nabla \phi\|_{\left(L^{2}\left(\Lambda^{\prime}\right)\right)^{9}},
$$

we get

$$
\left|I_{4}\right| \leq c_{M I} \varepsilon^{5 / 2}|f|\left(\|\nabla \phi\|_{\left(L^{2}\left(\tilde{\Omega}_{\varepsilon}\right)\right)^{9}}+\varepsilon|f|\right) .
$$

Using similar arguments and the fact that $k$ vanishes for $x_{3} \geq l_{3} / 2$, we get

$$
\begin{aligned}
& \left|I_{5}\right| \leq c \varepsilon^{3 / 2}|f|\|\nabla \phi\|_{\left(\left(L^{2}\left(\tilde{\Omega}_{\varepsilon}\right)\right)^{9}\right.}, \\
& \left|I_{6}\right| \leq c \varepsilon^{7 / 2}|f|\|\nabla \phi\|_{\left(\left(L^{2}\left(\tilde{\Omega}_{\varepsilon}\right)\right)^{9}\right.}, \\
& \left|I_{7}\right| \leq c_{M} \varepsilon^{5 / 2}|f|\|\nabla \phi\|_{\left(\left(L^{2}\left(\tilde{\Omega}_{\varepsilon}\right)\right)^{9}\right.} .
\end{aligned}
$$

From (37), the estimates (44), (46), (47), (49), (50), (51), and (52) give, for $\varepsilon$ small enough,

$$
\int_{\tilde{\Omega}_{\varepsilon}}|\nabla \phi|^{2} \leq c_{\Lambda I} \varepsilon^{3 / 2}|f|\left(\|\nabla \phi\|_{\left(\left(L^{2}\left(\tilde{\Omega}_{\varepsilon}\right)\right)^{9}\right.}+\varepsilon^{2}|f|\right) .
$$

This implies (35) if $\varepsilon$ is small enough.

8. Fixed point argument. It follows from (11) and (13) that $\bar{\Phi}_{\varepsilon}(t)$ possesses a limit $\bar{\Phi}_{\varepsilon \infty}$ as $t \rightarrow \infty$.

Lemma 8. There exists a positive number $c$ such that, for $\varepsilon<c$, the map $f \mapsto-\bar{\Phi}_{\varepsilon \infty}$ possesses a fixed point.

Proof. Given $M>0$, let us denote $B_{M I}=\left\{f \in \mathbb{R}^{3}: f_{3}=0\right.$ and $\left.|f| \leq M\right\}$.

Choice of $M$ such that $f \mapsto-\bar{\Phi}_{\varepsilon \infty}$ maps $B_{M I}$ into itself. Let $f \in B_{M I}$. By (13), $\bar{\Psi}_{\varepsilon}=\varepsilon\left(\bar{\Phi}_{\varepsilon}+f\right)$. Therefore,

$$
\left|\bar{\Phi}_{\varepsilon}(t)\right| \leq \frac{1}{\varepsilon}\left|\bar{\Psi}_{\varepsilon}(t)-\bar{\Psi}_{\varepsilon}(\varepsilon \bar{\eta})\right|+\left|\bar{\Phi}_{\varepsilon}(\varepsilon \bar{\eta})\right| .
$$

By (20) and (23),

$$
\left|\bar{\Psi}_{\varepsilon}(t)-\bar{\Psi}_{\varepsilon}(\varepsilon \bar{\eta})\right| \leq c \varepsilon^{3}(|g|+\varepsilon M) .
$$

On the other hand, by $(6), \Phi_{\varepsilon}\left(x^{\prime}, \eta_{\varepsilon}\left(x^{\prime}\right)\right)=-\frac{1}{l_{3}} \eta\left(x^{\prime} / \varepsilon\right)(g+\varepsilon f)$; thus

$$
\Phi_{\varepsilon}\left(x^{\prime}, \varepsilon \bar{\eta}\right)=\int_{\eta_{\varepsilon}\left(x^{\prime}\right)}^{\varepsilon \bar{\eta}} \partial_{z} \Phi_{\varepsilon}\left(x^{\prime}, z\right) d z-\frac{1}{l_{3}} \eta\left(\frac{x^{\prime}}{\varepsilon}\right)(g+\varepsilon f) .
$$

Therefore,

$$
\left|\bar{\Phi}_{\varepsilon}(\varepsilon \bar{\eta})\right| \leq \frac{1}{\varepsilon^{2} l_{1} l_{2}} \int_{S_{\varepsilon}} \int_{\eta_{\varepsilon}\left(x^{\prime}\right)}^{\varepsilon \bar{\eta}}\left|\partial_{z} \Phi_{\varepsilon}\left(x^{\prime}, z\right)\right| d z d x^{\prime}+\frac{\bar{\eta}}{l_{3}}(g+\varepsilon M) .
$$


Using the Cauchy-Schwarz inequality, $\partial_{z} \Phi_{\varepsilon}=\partial_{z} \Psi_{\varepsilon} / \varepsilon$, and (20), we get

$$
\left|\bar{\Phi}_{\varepsilon}(\varepsilon \bar{\eta})\right| \leq c\left((|g|+\varepsilon M)^{1 / 2}+(|g|+\varepsilon M)\right) .
$$

Using (53), (54), and (55) and passing to the limit as $t \rightarrow \infty$, we obtain

$$
\left|\bar{\Phi}_{\varepsilon \infty}\right| \leq c\left((|g|+\varepsilon M)^{1 / 2}+(|g|+\varepsilon M)\right) .
$$

Therefore, taking $M=2 c\left(|g|+|g|^{1 / 2}\right)$, there exists a positive number $c_{M}$ such that for $\varepsilon<c_{M I},\left|\bar{\Phi}_{\varepsilon \infty}\right| \leq M$. Then $-\bar{\Phi}_{\varepsilon \infty}$ belongs to $B_{M I}$, since its third component vanishes thanks to (13) and (22).

Choice of $\varepsilon$ such that $f \mapsto-\bar{\Phi}_{\varepsilon \infty}$ is a contractive map on $B_{M}$. Let $\left(\Phi_{\varepsilon}^{1}, \pi_{\varepsilon}^{1}\right)$ and $\left(\Phi_{\varepsilon}^{2}, \pi_{\varepsilon}^{2}\right)$ be the solutions, respectively, associated by $(6)$ and (13) with two vectors $f^{1}$ and $f^{2}$ of $B_{M}$. From (29) and (35) there exists a positive number $c_{M}$ such that, for any $t$,

$$
\left|\left(\overline{\Phi_{\varepsilon}^{2}}-\overline{\Phi_{\varepsilon}^{1}}\right)(t)\right|<c_{M} \varepsilon\left|f^{2}-f^{1}\right|
$$

Therefore, if $\varepsilon<1 / c_{M I}$,

$$
\left|\overline{\Phi_{\varepsilon \infty}^{2}}-\overline{\Phi_{\varepsilon \infty}^{1}}\right|<\alpha\left|f^{2}-f^{1}\right|, \quad \alpha<1 .
$$

Then the map $f \mapsto-\bar{\Phi}_{\varepsilon \infty}$ possesses a fixed point.

9. Exponential decay of the residue. Let us now choose $f=f_{\varepsilon}$ where $f_{\varepsilon}$ denotes the fixed point obtained in Lemma 8. Thanks to (13), we get $\bar{\Psi}_{\varepsilon \infty}=0$. Then (11) gives, for all $\alpha \in \mathbb{N}^{3}$ and $x_{3} \geq \varepsilon(\bar{\eta}+1)$,

$$
\left|\partial^{\alpha} \Psi_{\varepsilon}(x)\right|+\left|\partial^{\alpha} \xi_{\varepsilon}(x)\right| \leq c_{M}^{\prime} \exp \left(-c_{M}^{\prime \prime} \frac{x_{3}}{\varepsilon}\right) .
$$

To complete the proof of Theorem 1, according to the decomposition (9), it remains to get the exponential decay (14) of the residue $\left(\Xi_{\varepsilon}, \sigma_{\varepsilon}\right)$. At first, let us bound its $H^{1}$ norm, as follows.

LEMma 9. There exist positive numbers $c_{M I}, c_{M}^{\prime}$ and $c_{M I}^{\prime \prime}$ such that if $\varepsilon<c_{M I}$,

$$
\left\|\Xi_{\varepsilon}\right\|_{\left(H^{1}\left(\Omega_{\varepsilon}\right)\right)^{3}} \leq c_{\Lambda I}^{\prime} \exp \left(-\frac{c_{\Lambda I}^{\prime \prime}}{\varepsilon}\right) .
$$

Proof. In order to get a homogeneous boundary condition on the plane $\mathcal{P}$, let us define a divergence free extension $r$ of the trace of $-\Psi_{\varepsilon}$ on $\mathcal{P}$. At first, we define an extension $r^{1}$ in

$$
\omega=\left\{x: x^{\prime} \in S_{\varepsilon}, \frac{l_{3}}{2}<x_{3}<l_{3}\right\}
$$

by

$$
r^{1}\left(x^{\prime}, x_{3}\right)=-\Psi_{\varepsilon}\left(x^{\prime}, l_{3}\right) \frac{2 x_{3}-l_{3}}{l_{3}} .
$$

Clearly $r^{1} \in\left(H_{\mathrm{per}}^{1}(\omega)\right)^{3}$ and $\int_{\omega} \nabla \cdot r^{1}=0$. Then, see for instance [10], Theorem 3.1, p. 127 , there exists $r^{2} \in\left(H_{0}^{1}(\omega)\right)^{3}$ such that

$$
\nabla \cdot r^{2}=-\nabla \cdot r^{1} \text { in } \omega, \quad\left\|r^{2}\right\|_{\left(H^{1}(\omega)\right)^{3}} \leq c\left\|\nabla \cdot r^{1}\right\|_{\left(L^{2}(\omega)\right)} .
$$


Let us extend $r^{2}$ periodically in the $x^{\prime}$ variable. Then the function

$$
r= \begin{cases}r^{1}+r^{2} & \text { in } \omega, \\ 0 & \text { in } \Omega_{\varepsilon} \backslash \bar{\omega}\end{cases}
$$

belongs to $\left(H_{\text {per }}^{1}\left(\Omega_{\varepsilon}\right)\right)^{3}$ and satisfies $\nabla \cdot r=0$ and

$$
\|r\|_{\left(H^{1}\left(\Omega_{\varepsilon}\right)\right)^{3}} \leq c\left\|\Psi_{\varepsilon}\left(x^{\prime}, l_{3}\right)\right\|_{\left(H^{1}\left(S_{\varepsilon}\right)\right)^{3}} .
$$

Now let $\xi=\Xi_{\varepsilon}-r$ and $\sigma=\sigma_{\varepsilon}$. From (10), we get

$$
\begin{aligned}
& \xi \in\left(H_{\mathrm{per}}^{1}\left(\Omega_{\varepsilon}\right)\right)^{3}, \quad \sigma \in L_{\mathrm{per}}^{2}\left(\Omega_{\varepsilon}\right), \\
& -\nu \Delta \xi-\nu \Delta r+\xi \cdot \nabla \xi+r \cdot \nabla \xi+\xi \cdot \nabla r+r \cdot \nabla r \\
& \quad+\Psi_{\varepsilon} \cdot \nabla \xi+\Psi_{\varepsilon} \cdot \nabla r+\xi \cdot \nabla \Psi_{\varepsilon}+r \cdot \nabla \Psi_{\varepsilon}+\xi_{3} \frac{g+\varepsilon f_{\varepsilon}}{l_{3}}+r_{3} \frac{g+\varepsilon f_{\varepsilon}}{l_{3}} \\
& \quad+\left(\frac{x_{3}}{l_{3}}\left(g+\varepsilon f_{\varepsilon}\right)-\varepsilon f_{\varepsilon}\right) \cdot \nabla \xi+\left(\frac{x_{3}}{l_{3}}\left(g+\varepsilon f_{\varepsilon}\right)-\varepsilon f_{\varepsilon}\right) \cdot \nabla r+\nabla \sigma=0, \\
& \nabla \cdot \xi=0,\left.\quad \xi\right|_{\mathcal{P}}=0,\left.\quad \xi\right|_{\mathcal{R}_{\varepsilon}}=0 .
\end{aligned}
$$

Multiplying the equation by $\xi$ and integrating by parts, we get

$$
\begin{aligned}
\nu \int_{\Omega_{\varepsilon}}|\nabla \xi|^{2}= & -\nu \int_{\Omega_{\varepsilon}} \nabla r \cdot \nabla \xi-\int_{\Omega_{\varepsilon}} \xi \cdot \nabla r \cdot \xi-\int_{\Omega_{\varepsilon}} r \cdot \nabla r \cdot \xi-\int_{\Omega_{\varepsilon}} \Psi_{\varepsilon} \cdot \nabla r \cdot \xi \\
& -\int_{\Omega_{\varepsilon}} \xi \cdot \nabla \Psi_{\varepsilon} \cdot \xi-\int_{\Omega_{\varepsilon}} r \cdot \nabla \Psi_{\varepsilon} \cdot \xi-\int_{\Omega_{\varepsilon}} \xi_{3} \frac{g+\varepsilon f_{\varepsilon}}{l_{3}} \cdot \xi \\
& -\int_{\Omega_{\varepsilon}} r_{3} \frac{g+\varepsilon f_{\varepsilon}}{l_{3}} \cdot \xi-\int_{\Omega_{\varepsilon}}\left(\frac{x_{3}}{l_{3}}\left(g+\varepsilon f_{\varepsilon}\right)-\varepsilon f_{\varepsilon}\right) \cdot \nabla r \cdot \xi \\
= & J_{1}+\cdots+J_{9} .
\end{aligned}
$$

Let us now estimate $J_{1}, \ldots, J_{9}$ in terms of $\exp \left(-c_{M}^{\prime \prime} / \varepsilon\right)\|\nabla \xi\|_{\left(L^{2}\left(\Omega_{\varepsilon}\right)\right)^{9}}$ or of

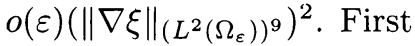

$$
\left|J_{1}\right| \leq \nu\|\nabla r\|_{\left(L^{2}\left(\Omega_{\varepsilon}\right)\right)^{9}}\|\nabla \xi\|_{\left(L^{2}\left(\Omega_{\varepsilon}\right)\right)^{9}} .
$$

By $(56)$ and (58),

$$
\|r\|_{\left(H^{1}\left(\Omega_{\varepsilon}\right)\right)^{3}} \leq c_{M I}^{\prime} \exp \left(-\frac{c_{M I}^{\prime \prime}}{\varepsilon}\right)
$$

and thus

$$
\left|J_{1}\right| \leq c_{M}^{\prime} \exp \left(-\frac{c_{M}^{\prime \prime}}{\varepsilon}\right)\|\nabla \xi\|_{\left(L^{2}\left(\Omega_{\varepsilon}\right)\right)^{9}} .
$$

For $J_{2}$, we remark that

$$
J_{2}=\int_{\Omega_{\varepsilon}} \xi \cdot \nabla r \cdot \xi=-\int_{\Omega_{\varepsilon}} \xi \cdot \nabla \xi \cdot r .
$$

The Hölder inequality gives

$$
\left|J_{2}\right| \leq c \varepsilon^{1 / 3}\|r\|_{\left(L^{6}\left(\Omega_{\varepsilon}\right)\right)^{3}}\|\xi\|_{\left(L^{6}\left(\Omega_{\varepsilon}\right)\right)^{3}}\|\nabla \xi\|_{\left(L^{2}\left(\Omega_{\varepsilon}\right)\right)^{9}} .
$$

Any function $\varphi \in\left(H^{1}\left(\Omega_{\varepsilon}\right)\right)^{3}$ that cancels on $R_{\varepsilon}$ satisfies

$$
\|\varphi\|_{\left(L^{6}\left(\Omega_{\varepsilon}\right)\right)^{3}} \leq c \varepsilon^{-2 / 3}\|\nabla \varphi\|_{\left(L^{2}\left(\Omega_{\varepsilon}\right)\right)^{9}} .
$$


This is proved in the same way as (43). Using in addition (60), we get

$$
\left|J_{2}\right| \leq c_{M}^{\prime} \exp \left(-\frac{c_{M}^{\prime \prime}}{\varepsilon}\right)\|\nabla \xi\|_{\left(L^{2}\left(\Omega_{\varepsilon}\right)\right)^{9}}^{2} .
$$

The same arguments lead to

$$
\left|J_{3}\right| \leq c_{M}^{\prime} \exp \left(-\frac{c_{M}^{\prime \prime}}{\varepsilon}\right)\|\nabla \xi\|_{\left(L^{2}\left(\Omega_{\varepsilon}\right)\right)^{9}} .
$$

For $J_{4}$, we remark that

$$
J_{4}=\int_{\Omega_{\varepsilon}} \Psi_{\varepsilon} \cdot \nabla r \cdot \xi=-\int_{\Omega_{\varepsilon}} \Psi_{\varepsilon} \cdot \nabla \xi \cdot r .
$$

The Hölder inequality gives

$$
\left|J_{4}\right| \leq c \varepsilon^{1 / 3}\|r\|_{\left(L^{6}\left(\Omega_{\varepsilon}\right)\right)^{3}}\left\|\Psi_{\varepsilon}\right\|_{\left(L^{6}\left(\Omega_{\varepsilon}\right)\right)^{3}}\|\nabla \xi\|_{\left(L^{2}\left(\Omega_{\varepsilon}\right)\right)^{9}} .
$$

Using the extension $h$ of $\Psi_{\varepsilon}$ defined by (25) and (62), we get

$$
\left\|\Psi_{\varepsilon}\right\|_{\left(L^{6}\left(\Omega_{\varepsilon}\right)\right)^{3}} \leq c \varepsilon^{-2 / 3}\left\|\nabla\left(\Psi_{\varepsilon}-h\right)\right\|_{\left(L^{2}\left(\Omega_{\varepsilon}\right)\right)^{9}}+\|h\|_{\left(L^{6}\left(\Omega_{\varepsilon}\right)\right)^{3}}
$$

and then

$$
\left\|\Psi_{\varepsilon}\right\|_{\left(L^{6}\left(\Omega_{\varepsilon}\right)\right)^{3}} \leq c \varepsilon^{-2 / 3}\left\|\nabla \Psi_{\varepsilon}\right\|_{\left(L^{2}\left(\Omega_{\varepsilon}\right)\right)^{9}}+c_{M} \varepsilon^{1 / 2} .
$$

Therefore, (20) and (60) give

$$
\left|J_{4}\right| \leq c_{M}^{\prime} \exp \left(-\frac{c_{M}^{\prime \prime}}{\varepsilon}\right)\|\nabla \xi\|_{\left(L^{2}\left(\Omega_{\varepsilon}\right)\right)^{9} .}
$$

The same arguments lead to

$$
\left|J_{5}\right| \leq c_{M}^{\prime} \varepsilon^{1 / 2}\|\nabla \xi\|_{\left(L^{2}\left(\Omega_{\varepsilon}\right)\right)^{9}}^{2}
$$

and

$$
\left|J_{6}\right| \leq c_{M}^{\prime} \exp \left(-\frac{c_{M}^{\prime \prime}}{\varepsilon}\right)\|\nabla \xi\|_{\left(L^{2}\left(\Omega_{\varepsilon}\right)\right)^{9}} .
$$

For $J_{7}$, we decompose the integral over $\Omega_{\varepsilon}$ into integrals over the domains

$$
\begin{aligned}
\omega^{\prime} & =\left\{x: x^{\prime} \in S_{\varepsilon}, \quad \eta_{\varepsilon}\left(x^{\prime}\right)<x_{3}<\varepsilon \bar{\eta}\right\}, \\
\omega^{\prime \prime} & =\left\{x: x^{\prime} \in S_{\varepsilon}, \quad \varepsilon \bar{\eta}<x_{3}<l_{3}\right\} .
\end{aligned}
$$

Since $\xi$ is divergence free and periodic, $\bar{\xi}=0$ in $\omega^{\prime \prime}$ and therefore

$$
J_{7}=\int_{\omega^{\prime}} \xi_{3} \frac{g+\varepsilon f_{\varepsilon}}{l_{3}} \cdot \xi+\int_{\omega^{\prime \prime}}\left(\xi_{3}-\bar{\xi}_{3}\right) \frac{g+\varepsilon f_{\varepsilon}}{l_{3}} \cdot(\xi-\bar{\xi}) .
$$

Using the Poincaré inequality (45) in $\omega^{\prime}$ (instead of $\Lambda^{\prime \prime}$ ) and the Poincaré-Wirtinger inequality (40) in $\omega^{\prime \prime}$ (instead of $\Theta^{\prime}$ ), we obtain

$$
\left|J_{7}\right| \leq c_{M}^{\prime} \varepsilon^{2}\left(\|\nabla \xi\|_{\left.\left(L^{2}\left(\Omega_{\varepsilon}\right)\right)^{9}\right)^{2}} .\right.
$$

Similarly,

$$
\left|J_{8}\right| \leq c_{M}^{\prime} \varepsilon^{2}\|\nabla r\|_{\left(L^{2}\left(\Omega_{\varepsilon}\right)\right)^{9}}\|\nabla \xi\|_{\left(L^{2}\left(\Omega_{\varepsilon}\right)\right)^{9}} .
$$


Using (60), this gives

$$
\left|J_{8}\right| \leq c_{\Lambda I}^{\prime} \exp \left(-\frac{c_{\Lambda I}^{\prime \prime}}{\varepsilon}\right)\|\nabla \xi\|_{\left(L^{2}\left(\Omega_{\varepsilon}\right)\right)^{9}} .
$$

For the last term in (59), we remark that

$$
\begin{aligned}
J_{9} & =\int_{\Omega_{\varepsilon}}\left(\frac{x_{3}}{l_{3}}\left(g+\varepsilon f_{\varepsilon}\right)-\varepsilon f_{\varepsilon}\right) \cdot \nabla r \cdot \xi \\
& =-\int_{\Omega_{\varepsilon}}\left(\frac{x_{3}}{l_{3}}\left(g+\varepsilon f_{\varepsilon}\right)-\varepsilon f_{\varepsilon}\right) \cdot \nabla \xi \cdot r .
\end{aligned}
$$

Thus

$$
\left|J_{9}\right| \leq c_{M I}^{\prime}\|r\|_{\left(L^{2}\left(\Omega_{\varepsilon}\right)\right)^{3}}\|\nabla \xi\|_{\left(L^{2}\left(\Omega_{\varepsilon}\right)\right)^{9}}
$$

and (60) gives

$$
\left|J_{9}\right| \leq c_{M}^{\prime} \exp \left(-\frac{c_{M I}^{\prime \prime}}{\varepsilon}\right)\|\nabla \xi\|_{\left(L^{2}\left(\Omega_{\varepsilon}\right)\right)^{9}}
$$

Now bounding the right-hand side of (59) with (61), (63). (64), (65), (66), (67), (68). (69), and (70) we prove the existence of a positive number $c_{M}$ such that for $\varepsilon<c_{N}$,

$$
\|\nabla \xi\|_{\left(L^{2}\left(\Omega_{\varepsilon}\right)\right)^{9}} \leq c_{\Lambda I}^{\prime} \exp \left(-\frac{c_{M I}^{\prime \prime}}{\varepsilon}\right) \text {. }
$$

Since $\Xi_{\varepsilon}=\xi+r$, using $(60)$, we get

$$
\left\|\Xi_{\varepsilon}\right\|_{\left(H^{1}\left(\Omega_{\varepsilon}\right)\right)^{3}} \leq c_{\Lambda I}^{\prime} \exp \left(-\frac{c_{M I}^{\prime \prime}}{\varepsilon}\right) .
$$

The proof of Lemma 9 is therefore complete.

Finally, the exponential decay (57) of the norm implies the pointwise decay (14) outside of a neighbourhood of the rough wall, that is, for $x_{3} \geq \varepsilon(\bar{\eta}+1)$. This has been done in Theorem 2 of [4] in the case of a half space. The adaptation of the proof to the present case of a strip $\left\{x: x^{\prime} \in \mathbb{R}^{2}, \varepsilon(\bar{\eta}+1)<x_{3}<l_{3}\right\}$ is elementary. Then we got $f_{\varepsilon}$ such that (11), (12), and (14) are satisfied. Thanks to the decomposition (9), this proves (4) and therefore Theorem 2.

\section{REFERENCES}

[1] Y. Achdou and O. Pironneau, Analysis of Wall laws. C. R. Acad. Sci. Paris, Série I, 320, 541-547 (1995)

[2] Y. Achdou, O. Pironneau, and F. Valentin. Effective boundary conditions for laminar flows over rough boundaries, J. Comput. Phys. 147, 187-218 (1998)

[3] Y. Amirat, D. Bresch, J. Lemoine. and J. Simon. A problem of exponential decay for Navier-Stokes equations arising in the analysis of rugosity. In Navier-Stokes equations and related nonlinear problems (Palanga, 1997), J. Pileckas, ed., H. Amann et al., VSP/TEV. Utrecht, 1998, pp. 1-14

[4] Y. Amirat, D. Bresch, J. Lemoine, and J. Simon. Existence of semi-periodic solutions of steady Navier-Stokes equations in a half space with an exponential decay at infinity, Rend. Sem. Mat. Univ. Padova 102, 341-365 (1999)

[5] Y. Amirat and J. Simon, Riblets and drag minimization, In Optimization Methods in Partial Differential Equations, S. Cox and I. Lasiccka, eds., Contemporary Mathematics, vol. 209, Amer. Math. Soc., Providence, RI, 1997, pp. 9-17 
[6] A. G. Belyaev, G. A. Chechkin, and A. L. Piatniski, Asymptotic behavior of the solutions of a boundary value problem in a perforated domain with oscillating boundary, Preprint, Université de Paris VI, 1998

[7] A. Carrau, Modèlisation numérique d'un écoulement sur paroi rugueuse, Thesis, Université de Bordeaux, 1992

[8] G. A. Chechkin, A. Friedman, and A. L. Piatniski, The boundary value problem in domains with very rapidly oscillating boundary, J. Math. Anal. Appl. 231, 213-234 (1999)

[9] J. Cousteix, Couche limite laminaire, Cepadues Editions, 1988

[10] G. P. Galdi, An Introduction to the Mathematical Theory of the Navier-Stokes Equations, Vols. 1 and 2, Springer-Verlag, New York, 1994

[11] O. A. Ladyshenskaya and V. A. Solonnikov, Determination of solutions of boundary value problems for steady-state Stokes and Navier-Stokes equations in domains having an unbounded Dirichlet integral, J. Soviet Math. 21, 728-761 (1983)

[12] R. Lewandowski, Analyse mathématique et océanographie, Masson, 1997

[13] J.-L. Lions, Quelques méthodes de résolutions des problèmes aux limites non linéaires, Dunod, Paris, 1969

[14] J.-L. Lions, Perturbations singulières dans les problèmes aux limites et en contrôle optimal, Lecture Notes in Mathematics 323, Springer-Verlag, New York, 1973

[15] J.-L. Lions, Some Methods in the Mathematical Analysis of Systems and Their Control, Science Press, Beijing, China, Gordon and Breach, Inc. New York, 1981 